\title{
Neurotropic Effect of Brain-Derived Neurotrophic Factor at Different Stages of Dissociated Hippocampal Cultures Development in vitro
}

DOI: 10.17691/stm2015.7.3.06

Received March 27, 2015

T.A. Mishchenko, Junior Researcher, Molecular and Cell Technologies Department, Central Research Laboratory'

Junior Researcher, Laboratory for Pharmacological Properties of Neurotropic Drug Research,

Nizhny Novgorod Neuroscience Centre, Institute of Biology and Biomedicine²;

M.V. Vedunova, PhD, Head of the Laboratory for Neuroprotection Methods Development, Nizhny Novgorod Neuroscience Centre, Institute of Biology and Biomedicine2; Senior Researcher, Biochemistry Department, Central Research Laboratory';

E.V. Mitroshina, Researcher, Department of Molecular and Cell Technologies, Central Research Laboratory'; Researcher, Laboratory for Neuroprotection Methods Development, Nizhny Novgorod Neuroscience Centre, Institute of Biology and Biomedicine;

A.S. Pimashkin, PhD, Assistant, Neurodynamics and Neurobiology Department, Biological Faculty;

I.V. Mukhina, DSc, Professor, Head of the Central Research Laboratory'; Head of the Department of Normal Physiology named after N.Y. Belenkov'; Professor, Neurodynamics and Neurobiology Department, Institute of Biology and Biomedicine ${ }^{2}$

${ }^{1}$ Nizhny Novgorod State Medical Academy, 10/1 Minin and Pozharsky Square, Nizhny Novgorod, 603005,

Russian Federation;

${ }^{2}$ Lobachevsky State University of Nizhni Novgorod, 23 Prospect Gagarina, Nizhny Novgorod, 603950,

Russian Federation

The aim of the investigation was to evaluate the neurotropic effect of brain-derived neurotrophic factor (BDNF) on the spontaneous bioelectrical activity of hippocampal neural networks according to the stage of their development in vitro.

Materials and Methods. The studies were performed using hippocampal cells dissociated from C57BL/6 mice embryos (E18) and cultured on multielectrode arrays consisting of 64 electrodes (MED64, Alpha Med Science, Japan). BDNF was applied to the culture medium in concentrations $0.1,1.0$, and $10 \mathrm{ng} / \mathrm{ml}$ on days 7,14 and 21 of the culture development in vitro.

Spontaneous bioelectrical activity of dissociated hippocampal cultures was recorded within 10 min before the BDNF application. After the neurotrophin addition, the activity was recorded continuously for $35 \mathrm{~min}$. The 10-minute recordings was repeated at 2 and $24 \mathrm{~h}$ after the BDNF application.

Results. The experiments revealed that BDNF modulates the spontaneous bioelectrical activity of dissociated hippocampal cultures, starting from day 14 of culture development in vitro. The effect is manifested as increased network burst duration and in the restructuring of the pattern of spontaneous network activity without any changes in the number of spikes in a burst. BDNF neurotropic action was observed between 10 and 15 min after application with a validity period not less than $2 \mathrm{~h}$.

Conclusion. BDNF $(0.1 ; 1.0 ; 10 \mathrm{ng} / \mathrm{ml})$ application has a transient neurotropic effect on the spontaneous bioelectrical activity of mature neural networks starting from day 14 of dissociated hippocampal cultures development in vitro. Investigation the mechanisms of BDNF participation in synaptic transmission clarifies the role this neurotrophin in such functions of the central nervous system, as learning and memory processes.

Key words: brain-derived neurotrophic factor; BDNF; dissociated hippocampal cultures; multielectrode arrays.

The study of the mechanisms of biological processes regulation observed in the central nervous system (CNS) is one of the topical issues in modern biology and medicine. Neurotrophins are considered as the most important regulatory proteins involved in the maintaining of human and animal vital processes [1]. Brain-derived neurotrophic factor (BDNF) is a key representative of this protein's family, whose functions directly relate to the work of CNS. This neurotrophin plays a major role at perinatal stage of development participating in neurogenesis and providing active differentiation and proliferation of neurons with different phenotypes and localizations. However the effect of BDNF on CNS functioning in the postnatal period is of special interest [2]. Recent studies [3] showed the BDNF possibility to modulate synaptic transmission in the hippocampus and in some parts of the cortex. BDNF interaction with the high affinity tropomyosin-related kinase B receptor (TrkB)

For contacts: Mishchenko Tatiana Alexandrovna, e-mail: saHarnova87@mail.ru 
launches several signal mechanisms providing the effect of this neurotrophin on presynaptic and postsynaptic transmission. BDNF is capable of activating potentialdependent sodium and potassium channels, and increasing the level of GABAA-receptors expression as well as NR1- and NR2B-subuntis of NMDA-receptors phosphorylation thereby affecting the efficiency of synaptic transmission. Furthermore, BDNF takes part in the formation of synaptic plasticity and in such processes as learning and memory $[4,5]$. However due to the lack of knowledge about the role and mechanisms of BDNF effect on neural networks functioning at different stages of their development or about its participation in homeostatic plasticity, further investigation of these issues is required.

The aim of the investigation was to evaluate the neurotropic effect of brain-derived neurotrophic factor on the spontaneous bioelectrical activity of hippocampal neural networks according to the stage of their development in vitro.

\section{Materials and Methods}

Cell culture. Hippocampal cells were dissociated from embryonic C57BL/6 mice (E18). The main rules of keeping and caring for experimental animals corresponded to the norms provided in the order of the Ministry of the Russian Federation No.267 dated 19 June 2003 "About the Rules of Good Laboratory Practice in the Russian Federation". To record the main electrophysiological parameters of the neural networks activity the cells were cultivated on multielectrode arrays MED64 (Alfa Med Science, Japan).

Enzymatic dissociation of the embryonic hippocampal cells was performed by treating them with $0.25 \%$ trypsin (Gibco, USA). Cultivation of the primary cultures was performed in Neurobasal ${ }^{\mathrm{TM}}$ medium (Invitrogen, USA) containing the bioactive additives: B27 (Invitrogen, USA), L-glutamine (Invitrogen, USA), fetal calf serum (PanEko, Russia), according to the previously developed protocol [6] during 30 days in vitro. Before cell cultivation the multielectrode arrays pretreated with the positively charged hydrophilic substance - polyethyleneimine (Sigma, Germany) to increase cell adhesion to their surface. The initial density of cells on the multielectrode arrays was approximately $9 \cdot 10^{3}$ cells $/ \mathrm{mm}^{2}$. The viability of the cells was maintained under constant conditions of $35.5^{\circ} \mathrm{C}$ and $5 \% \mathrm{CO}_{2}$ at saturating humidity in a cell culture incubator (MCO-18AIC, Sanyo, Japan) [7].

Scheme of the experiment. BDNF was applied to the culture medium on days 7,14 and 21 of culture development in vitro. All cultures were divided into the following experimental groups: 1) BDNF $0.1 \mathrm{ng} / \mathrm{ml}$; 2) BDNF $1 \mathrm{ng} / \mathrm{ml}$; 3) BDNF $10 \mathrm{ng} / \mathrm{ml}$. Corresponding concentrations of albumin solution in phosphate buffered saline (PBS) was used as a controls.

The spontaneous bioelectrical activity of dissociated hippocampal cultures was recorded 10 min before BDNF application. After neurotrophin addition the activity was recorded continuously over the next $35 \mathrm{~min}$. The 10minute recordings were repeated at 2 and at $24 \mathrm{~h}$ after BDNF application (Figure 1).

For obtaining the data and their primary analysis Conductor $^{\mathrm{TM}}$ (Alpha Med Science, Japan) software was used. Analysis of the neural network activity was performed using the original pack of algorithms MEAMAN (Certificate of State Registration of PC Software No.2012611190), developed in the custommade software MATLAB.

Spike (extracellular potentials) detection was performed according to the following algorithm: a limit of $8 \sigma$ was selected, where $\sigma$ is the mean square value. If the amplitude of spontaneous activity increased over this limit, it was considered to be a spike, while, if activity was not thus increased, it was considered to be noise and was not taken into account when the data were analyzed. The following parameters of the spontaneous neural network activity were investigated: the number of small network bursts, the number of spikes in a burst, the duration of small network burst. The criterion of a small network burst was the presence of spikes at least at 4 different electrodes of the multielectrode array with inter-spike intervals not more than $100 \mathrm{~ms}$ [8].

Analysis of neural network activation patterns. Additionally, to characterize the functional structure of the network activity an analysis of spikes sequence inside each burst was performed. To investigate the functional characteristics of all the recorded cells the following analysis of activation patterns was carried out. For each burst a 64-dimensional activation pattern was determined - from the time of the first spike on each electrode after the start of the burst.

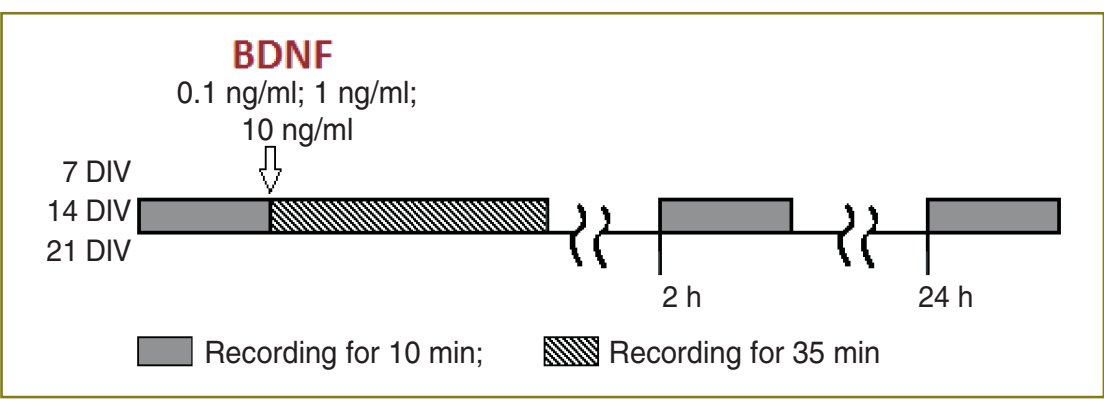

Figure 1. Scheme of spontaneous bioelectrical activity recordings from dissociated hippocampal cultures 
Statistical data analysis were performed using nonparametric Mann-Whitney criterion implemented in the Sigma Plot 11.0 software program (Systat Software, Inc.). All quantified data are presented as the mean \pm standard error of the mean values. Differences between groups were considered significant if the corresponding $p$ value was less than 0.05 .

Results. Investigation of BDNF neurotropic effect

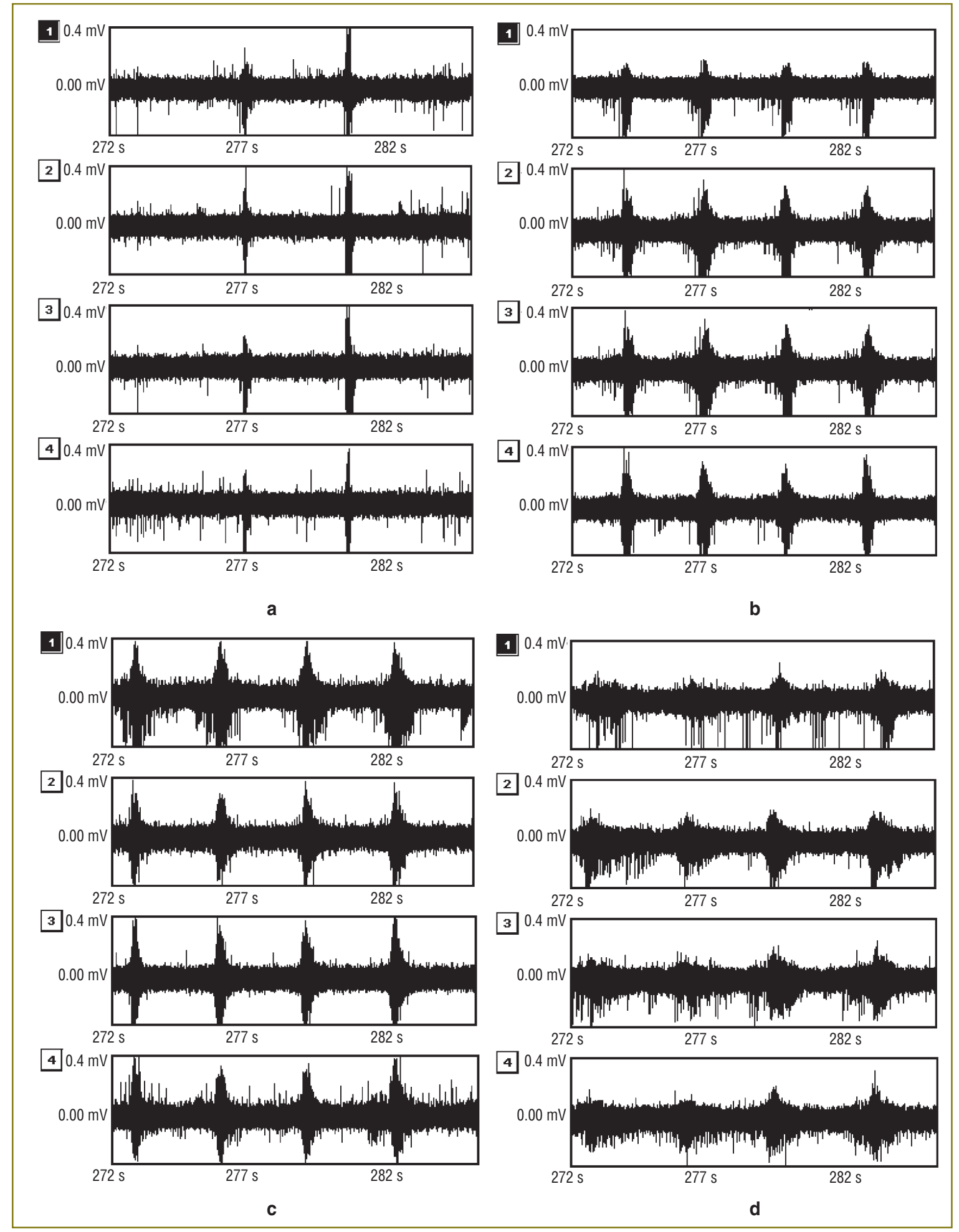

Figure 2. Spike recording from 4 (1-4) electrodes of the MED64 probe (Alfa MED Science, Japan) on day 7 of culture development in vitro: (a) before BDNF (1.0 ng/ml) application; (b) $20 \mathrm{~min}$ after BDNF (1.0 ng/ml) application; (c) $2 \mathrm{~h}$ after BDNF (1.0 ng/ml) application; (d) $24 \mathrm{~h}$ after BDNF (1.0 $\mathrm{ng} / \mathrm{ml})$ application 
on the functional bioelectrical activity of dissociated hippocampal cells was performed on days 7, 14 and 21 in vitro. According to the previously obtained data these developmental terms represent the key stages of neural networks formation in primary cultures in vitro [9-12]. The addition of albumin in PBS in different concentrations did not cause any statistically significant changes in the spontaneous bioelectrical activity parameters at any stages of primary cultures development, so this justified the averaging of the control group values for each part of the experiment.

It was shown that in response to BDNF application $(0.1$ and $1.0 \mathrm{ng} / \mathrm{ml}$ ) on day 7 of culture development in vitro the spontaneous bioelectrical activity did not significantly change in any of the studied parameters (the number of small network bursts, the number of

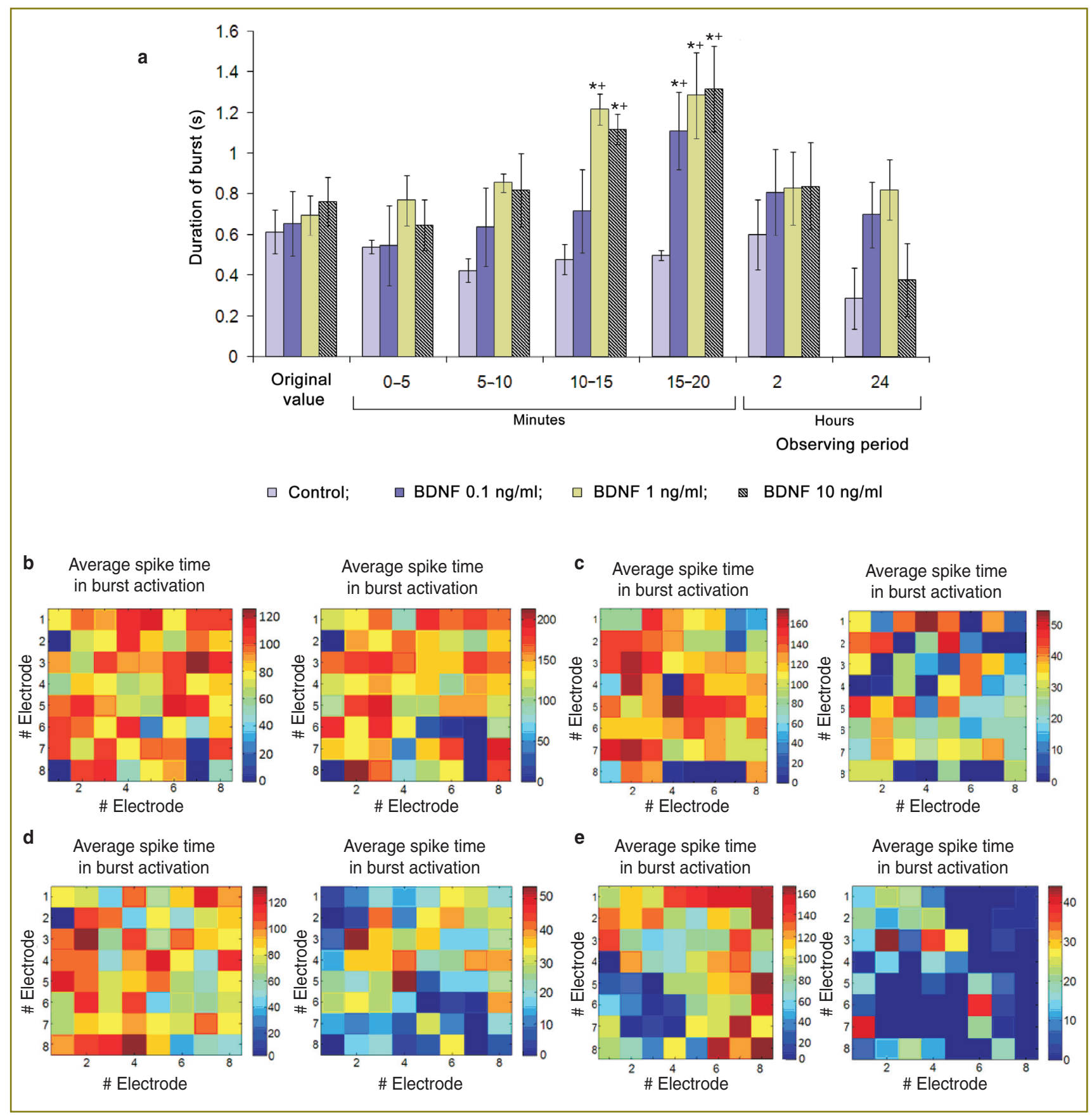

Figure 3. Effect of BDNF on the duration of small neural network burst on day 14 of culture development in vitro (a); * statistical significance with the original value; + with the control group, $\mathrm{p}<0.05$ (Mann-Whitney criterion); (b)-(e) the patterns of spontaneous bioelectrical activity of primary hippocampal cultures. On the left - before BDNF application, on the right $-24 \mathrm{~h}$ after BDNF application: (b) control; (c) BDNF $0.1 \mathrm{ng} / \mathrm{ml}$; (d) BDNF $1.0 \mathrm{ng} / \mathrm{ml}$; (e) BDNF $10 \mathrm{ng} / \mathrm{ml}$. Color diagram — time of spike occurrence in the small neural network, recorded from the electrodes, $\mathrm{ms}$ 
spikes per burst, the duration of the small network burst). Nevertheless a synchronization of neurons activity responsible for the network burst formation after BDNF injection was observed (Figure 2 (b), (c)). This effect was registered over $24 \mathrm{~h}$ (Figure 2 (d)).

Next we noted the changes in spontaneous bioelectrical activity in response to BDNF application on day 14 of culture development in vitro. The most significant events were identified in the duration of small network burst. In the period from 15 to $20 \mathrm{~min}$ after BDNF $0.1 \mathrm{ng} / \mathrm{ml}$ addition to the culture medium this parameter was significantly increased $(p<0.05)$ compared to the initial level, on average, by $70 \%$ (from $0.65 \pm 0.16$ to $1.11 \pm 0.19 \mathrm{~s}$ ) as well as in comparison to the activity of the control cultures $(p<0.05)$. However, the neurotropic effect was observed not less than $20 \mathrm{~min}$. The spontaneous bioelectrical activity returned to its original values and did not differ from the control cultures activity $2 \mathrm{~h}$ after BDNF application (Figure $3(\mathrm{a})$ ).

Similar dynamics for the changes in spontaneous bioelectrical activity were shown in the group of cultures where BDNF had been injected at $1 \mathrm{ng} / \mathrm{ml}$ concentration. The effect from neurotrophin action in this case was more evident than in the "BDNF $0.1 \mathrm{ng} / \mathrm{ml}$ " group. In 10-15 min, the duration of small network burst had a statistically higher value $(p<0.05)$ in comparison with the initial activity and $0.1 \mathrm{ng} / \mathrm{ml}$ BDNF application. At $20^{\text {th }}$ minute of the recording burst duration, on average $(p<0.05)$, exceeded the initial values by $83 \%$ (from $0.698 \pm 0.09$ to $1.286 \pm 0.21 \mathrm{~s}$ ), but it was equal with the values in the group with lower BDNF concentration. At 2 and $24 \mathrm{~h}$ after the neurotrophin addition the spontaneous bioelectrical activity in the group of cultures with BDNF $1.0 \mathrm{ng} / \mathrm{ml}$ application returned to the original values.

Analysis of the spontaneous bioelectrical activity parameters in experimental group with BDNF $10 \mathrm{ng} / \mathrm{ml}$ application (Figure 2 (a)) showed that by the $10-15^{\text {th }}$ minute of recording the duration of small network burst significantly increased $(p<0.05)$ compared to its initial value and with the value of the group with BDNF $0.1 \mathrm{ng} / \mathrm{ml}$ addition. The significant differences from "BDNF $1.0 \mathrm{ng} / \mathrm{ml}$ " group of cultures did not observe.

Thus, it was noticed the neurotropic effect of BDNF at 1.0 and $10 \mathrm{ng} / \mathrm{ml}$ concentrations and was not revealed pronounced effect of neurotrophic factor for $0.1 \mathrm{ng} / \mathrm{ml}$. Neurotrophin action lasted for a short time, and the activity of neural networks had returned to their initial level $2 \mathrm{~h}$ after BDNF injection.

Furthermore, the application of BDNF in different concentrations caused the modifications in the functional characteristics of network bursts, reflecting the individual structure of the neural networks of dissociated hippocampal cultures (Figure $3(\mathrm{~b})-(\mathrm{e})$ ). It is assumed that BDNF has a neurotropic effect associated with an elevation of synaptic transmission efficiency, which cause an increase the number of neurons with an activation time more than 50 ms. Such synchronization of neurons in the network was observed $24 \mathrm{~h}$ after BDNF application. It should be noted that the degree of spikes synchronization depended on neurotrophin concentration. The greatest effect was found in the group of cultures "BDNF $10 \mathrm{ng} / \mathrm{ml}$ ", where the time of spike occurrence in the network burst on the majority of electrodes was not more than $15 \mathrm{~ms}$.

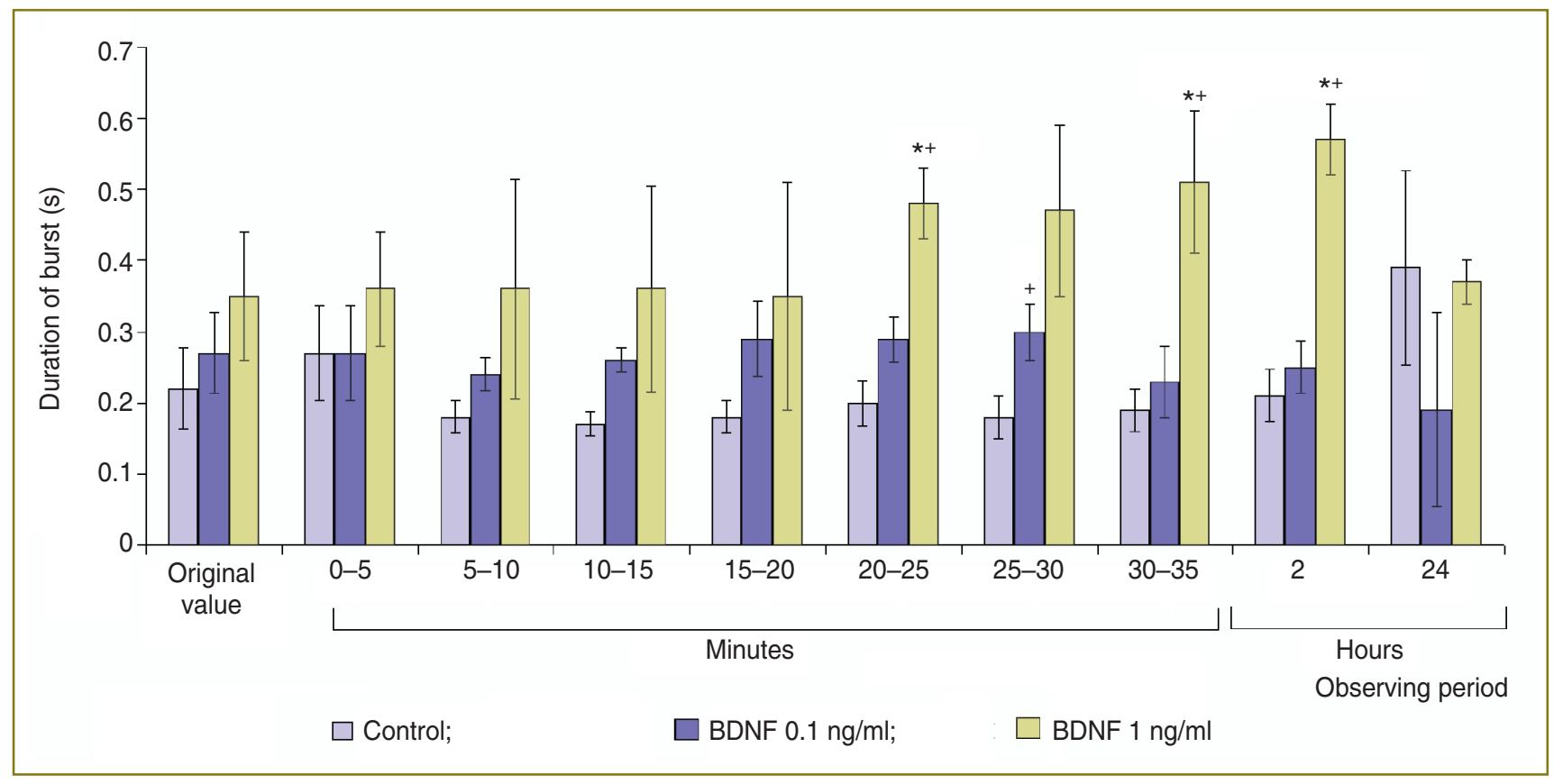

Figure 4. Effect of BDNF on the duration of small neural network burst on day 21 of culture development in vitro; * statistical significance with the original value; ${ }^{+}$with the control group, $p<0.05$ (Mann-Whitney criterion) 
The experiments performed on day 21 of culture development in vitro showed that BDNF $1.0 \mathrm{ng} / \mathrm{ml}$ application leads to the activation of spontaneous bioelectrical activity of primary hippocampal cultures. Significant changes in the duration of small network burst were recorded $20 \mathrm{~min}$ after BDNF addition. The application of lower BDNF concentrations $(0.1 \mathrm{ng} / \mathrm{ml})$ did not significantly change the bioelectrical activity parameters of primary cultures (Figure 4).

It was found a statistically significant increase $(p<0.05)$ in the duration of small network burst 20 min after BDNF $1.0 \mathrm{ng} / \mathrm{ml}$ injection in comparison to the initial activity as well as the activity of control cultures, lasting throughout the following $2 \mathrm{~h}$ (the control values were exceeded, on average, by $62 \%$ ). On the other hand the effect was transient and the burst duration did not differ from the initial and control values by $24 \mathrm{~h}$ after BDNF addition.

The analysis of the functional characteristics of the network bursts reflecting the individual structure of a neural network showed that the addition of a control solution (albumin in PBS) did not affect on the activation pattern of network bursts of primary cultures. In contrast, BDNF application at 0.1 and $1.0 \mathrm{ng} / \mathrm{ml}$ concentrations caused a reduction in the time of the first spike occurrence which testifies to increased synchronization of neuron activity. This effect was preserved during $24 \mathrm{~h}$ after neurotrophin injection, as well as on day 14 of dissociated hippocampal cultures development.

Discussion. The modulation of spontaneous bioelectrical activity of dissociated hippocampal cultures by brain-derived neurotrophic factor was shown. The changes in the spontaneous neural networks activity were expressed as an increase in the duration of small network burst and a reduction in the time for the first spike occurrence, which leads to restructuring the pattern of network activity and the whole alterations in functional network characteristics. The manifestation of the registered modifications depended on BDNF concentration and directly related to the stage of culture development in vitro.

BDNF application on day 7 of culture development in vitro did not affect the spontaneous bioelectrical activity of dissociated hippocampal cultures, whereas on days 14 and 21 of growth the neural network activity was modulated. The response to the neurotrophin injection was developed within 10-20 min and expressed as an increase in the duration of small network burst. Moreover, a typical manifestation of the neurotrophic effect of BDNF was an elevation the efficiency of synaptic transmission in the hippocampal neural network and the enhancement of synchronization of the neurons in network activity.

The neurotropic effect supposed to be connected with metabolic processes mediated by BDNF and TrkB receptor interactions with the subsequent launching of signaling cascades $[3,13-15]$. To implement these reactions, mature synaptic contacts in the neural network are required $[4,5,16]$. This explains the lack of changes in the spontaneous bioelectrical activity when the neurotrophin was added on day 7 of dissociated hippocampal cultures development, because, this period is characterized as a stage of neural network formation with prevalence of electrical synapses and absence of mature chemical synaptic contacts [9-12]. At the later stages of primary hippocampal cultures development there is presence of formed neural networks with chemical synapses in their structure, providing plasticity in dissociated cultures $[9,10]$ and being necessary for the realization the neurotropic effect of BDNF.

Thus, BDNF may be considered as neuromodulator of spontaneous bioelectrical activity of neural networks in dissociated hippocampal cultures. Investigation the mechanisms of BDNF participation in synaptic transmission clarifies the role of this neurotrophin in such functions of the central nervous system as learning and memory.

Conclusion. Brain-derived neurotrophic factor in concentrations of $0.1,1.0$ and $10 \mathrm{ng} / \mathrm{ml}$ has a transient neurotrophic effect on the spontaneous bioelectrical activity of mature neural networks starting from day 14 of primary hippocampal cultures development in vitro.

Study Finding. The study was supported by grants from the Russian Foundation for Basic Research No.1304-01871, No.13-04-12067, No.14-04-31601 and partially supported by a further grant (from the agreement dated August 27, 2013 No.02.B.49.21.0003 between the Ministry of Education of the Russian Federation and the Lobachevsky State University of Nizhni Novgorod). The publication has been prepared as part of the state project "The Provision of Scientific Research".

Conflicts of Interest. The authors have no conflict of interest.

\section{References}

1. Gomazkov O.A. Neyrotroficheskaya regulyatsiya i stvolovye kletki mozga [Neurotrophic regulation and brain stem cells]. Moscow: Ikar; 2006; 331 p.

2. Martin J.L., Finsterwald C. Cooperation between BDNF and glutamate in the regulation of synaptic transmission and neuronal development. Commun Integr Biol 2011; 4(1): 14-16, http://dx.doi.org/10.4161/cib.13761.

3. Rose C.R., Blum R., Kafitz K.W., Kovalchuk Y., Konnerth A. From modulator to mediator: rapid effects of BDNF on ion channels. BioEssays 2004; 26(11): 1185-1194, http:// dx.doi.org/10.1002/bies.20118.

4. Aptowicz C.O., Kunkler P.E., Kraig R.P. Homeostatic plasticity in hippocampal slice cultures involves changes in voltage-gated $\mathrm{Na}^{+}$channel expression. Brain Res 2004; 998(2): 115-163, http://dx.doi.org/10.1016/j.brainres.2003.11.035.

5. Cunha C., Brambilla R., Tomas K.L. A simple role for BDNF in learning and memory? Front Mol Neurosci 2010; 3: 1, http://dx.doi.org/10.3389/neuro.02.001.2010.

6. Mukhina I.V., Kazantsev V.B., Khaspeckov L.G., Zakharov Yu.N., Vedunova M.V., Mitroshina E.V., Korotchenko S.A., Koryagina E.A. Multielectrode matrices - 
new possibilities in investigation of the neuronal network plasticity. Sovremennye tehnologii v medicine 2009; 1: 8-15.

7. Vedunova M., Sakharnova T., Mitroshina E., Perminova M., Pimashkin A., Zakharov Y., Dityatev A., Mukhina I. Seizure-like activity in hyaluronidase-treated dissociated hippocampal cultures. Front Cell Neurosci 2013; 7(149), http://dx.doi.org/10.3389/fncel.2013.00149.

8. Pimashkin A., Kastalskiy I., Simonov A., Koryagina E., Mukhina I., Kazantsev V. Spiking signatures of spontaneous activity bursts in hippocampal cultures. Front Comput Neurosci 2011; 5(46), http://dx.doi.org/10.3389/fncom.2011.00046.

9. Shirokova O.M., Frumkina L.E., Vedunova M.V., Mitroshina E.V., Zakharov Y.N., Khaspekov L.G., Mukhina I.V. Morphofunctional patterns of neuronal network developing in dissociated hippocampal cell cultures. Sovremennye tehnologii $v$ medicine 2013; 5(2): 6-13.

10. Agrba E.A., Mukhina I.V. Spatio-temporal characteristics of neuronal network activity of primary hippocampal cultures. Vestnik Nizhegorodskogo universiteta im. N.I. Lobachevskogo 2013; 4(1): 139-144.

11. Mitroshina E.V., Vedunova M.V., Shirokova O.M., Zakharov Yu.N., Kalintseva Ya.I., Mukhina I.V. Assessment of functional state dynamics of dissociated hippocampal cell culture in vitro. Vestnik Nizhegorodskogo universiteta im. N.I. Lobachevskogo 2011; 2(2): 283-286.
12. Gladkov A.A., Vedunova M.V., Korotchenko S.A., Zakharov Yu.N., Balashova A.N., Mukhina I.V. Development of spatiotemporal structure of hippocampal neural network in vitro. Vestnik Nizhegorodskogo universiteta im. N.I. Lobachevskogo 2011; 2(2): 243-248.

13. Caldeira M.V., Melo C.V., Pereira D.B., Carvalho R.F., Carvalho A.L., Duarte C.B. BDNF regulates the expression and traffic of NMDA receptors in cultured hippocampal neurons. Mol Cell Neurosci 2007; 35(2): 208-219, http://dx.doi.org/10.1016/ j.mcn.2007.02.019.

14. Porcher C., Hatchett C., Longbottom R.E., McAinch K., Sihra T.S., Moss S.J., Thomson A.M., Jovanovic J.N. Positive feedback regulation between gamma-aminobutyric acid type $A$ $(G A B A(A))$ receptor signaling and brain-derived neurotrophic factor (BDNF) release in developing neurons. $J$ Biol Chem 2011; 286(24): 21667-21677, http://dx.doi.org/10.1074/jbc. M110.201582.

15. Patapoutian A., Reichardt L.F. Trk receptors: mediators of neurotrophin action. Curr Opin Neurobiol 2001; 11(3): 272-280, http://dx.doi.org/10.1016/S0959-4388(00) 00208-7.

16. Sakharnova T.A., Vedunova M.V., Mukhina I.V. Brainderived neurotrophic factor (BDNF) and its role in the functioning of the central nervous system. Neurochem $J$ 2012; 6(4): 251259, http://dx.doi.org/10.1134/s1819712412030129. 